\title{
BAlloon versus Stenting in severe Ischaemia of the Leg-3 (BASIL-3): study protocol for a randomised controlled trial
}

\author{
Benjamin D. Hunt ${ }^{1 *}$, Matthew A. Popplewell², Huw Davies², Lewis Meecham², Hugh Jarrett ${ }^{1}$, Gareth Bate², \\ Margaret Grant ${ }^{1}$, Smitaa Patel ${ }^{1}$, Catherine Hewitt ${ }^{1}$, Lazaros Andronis ${ }^{3}$, Jonathan J. Deeks ${ }^{1}$, Andrew Bradbury ${ }^{2}$ \\ and on behalf of the BASIL-3 Collaborative Group
}

\begin{abstract}
Background: Severe limb ischaemia (SLI) is defined as the presence of rest pain and/or tissue loss secondary to lower extremity atherosclerotic peripheral arterial disease. The superficial femoral and popliteal arteries are the most commonly diseased vessels in such patients and are being increasingly treated using endovascular revascularisation techniques. However, it is currently unknown whether drug-eluting stents and drug-coated balloons confer additional clinical benefits over more established techniques using plain balloons and bare metal stents, or whether they represent a cost-effective use of NHS resources.

Methods: The BASIL-3 trial is a UK National Institute for Health Research, Health Technology Assessment Programme-funded, multicentre, randomised controlled trial (RCT) comparing the clinical and cost-effectiveness of plain balloon angioplasty with or without bail-out bare metal stenting, drug-coated balloon angioplasty with or without bail-out bare metal stenting, and primary stenting with drug-eluting stents for SLI secondary to femoropopliteal disease. Patients with 'multilevel' disease may receive aorto-iliac and/or infrapopliteal treatments concurrently with their randomised femoro-popliteal intervention. The primary clinical outcome is amputation-free survival defined as the time to major (above the ankle) amputation of the index limb or death from any cause. The primary outcome for the economic analysis is cost per quality-adjusted life year. Secondary outcome measures include overall survival, major adverse limb events, major adverse cardiac events, relief of ischaemic pain, healing of tissue loss, and quality of life. The required sample size has been calculated at 861 participants (287 on each arm). These patients will be recruited over 3 years and followed-up for between 2 and 5 years.

Discussion: BASIL-3 is a pragmatic RCT designed to reflect current UK clinical practice. The results will inform decision-making regarding the appropriateness of funding the use of drug-coated balloons and drug-eluting stents, by the NHS, for the management of SLI due to femoro-popliteal disease.
\end{abstract}

Trial registration: ISRCTN Registry, identifier: ISRCTN14469736. Registered on 22 October 2015.

Keywords: Severe limb ischaemia, Critical limb ischaemia, Endovascular treatment, Angioplasty, Stent, Drug-coated balloon, Drug-eluting stent, Diabetes, Cost-effectiveness

\footnotetext{
* Correspondence: b.hunt@bham.ac.uk

${ }^{1}$ Birmingham Clinical Trials Unit, Institute of Applied Health Research, College

of Medical and Dental Sciences, Public Health Building, University of

Birmingham, Birmingham B15 2TT, UK

Full list of author information is available at the end of the article
} 


\section{Background}

Peripheral arterial disease (PAD) is caused by a gradual build-up of atheroma within the arterial wall leading to the lumen becoming stenosed (narrowed) and ultimately occluded. Modifiable risk factors for the development of PAD include cigarette smoking, hypertension, dyslipidaemia, diabetes mellitus (DM), chronic kidney disease (CKD), increased Body Mass Index, and sedentary lifestyle. PAD is also associated with increasing age and male sex, and there may be a familial predisposition in some cases [1]. PAD presents as a continuum from asymptomatic disease to intermittent claudication (pain in the leg on walking due to ischaemia, relieved by rest) through to severe (or critical) limb ischaemia (SLI) characterised by [2]:

1. Severe and constant ischaemic pain which is experienced at rest, especially at night, usually in the forefoot

2. Tissue loss in the form of ulceration or gangrene, typically this starts with a minor foot injury which fails to heal and/or becomes infected

In the Western world, it has been estimated that there will be between 500 and 1000 new cases of SLI each year per million general population although the current epidemiology of SLI in the United Kingdom (UK) is not well defined [3]. The incidence of SLI is increasing globally as a consequence of ageing populations and the increasing prevalence of modifiable risk factors, especially tobacco consumption and DM [4]. SLI patients tend to have multiple comorbidities, to be at high cardiovascular risk, and untreated have up to $50 \%$ mortality within 1 year of diagnosis [5]. Failure to promptly revascularise in SLI patients is associated with greater use of health care services and poorer outcomes including major amputation and death [6].

SLI is currently managed by the following methods $[5,6]$ :

1. Best medical therapy, comprising antiplatelet medication, lipid modification, optimal diabetic/ blood pressure control, analgesia, and foot and wound care

2. Surgical revascularisation, usually with autologous vein bypass and/or endarterectomy

3. Endovascular revascularisation, performed under local anaesthetic and with balloon catheters and/ or stents

4. Primary amputation, where the limb is considered to be unsalvageable or the patient is either unwilling or unable to undergo revascularisation

5. End of life care for those deemed unfit for revascularisation or primary amputation
Endovascular revascularisation techniques are increasingly being used as a first-line treatment for SLI, especially when a suitable vein is not available for bypass. Traditionally, best endovascular treatment consists of plain balloon angioplasty (PBA) with so-called 'bail-out' bare metal stenting (BMS) when PBA alone has been technically unsuccessful. More recently, drug-coated balloons (DCB) and drug-eluting stents (DES) have entered the market. These devices are designed to deliver various drugs (most commonly paclitaxel) locally to the lesion with the aim of reducing the incidence of restenosis following angioplasty or stenting $[7,8]$. However, evidence of clinical efficacy is lacking and there are unknown NHS cost implications for their use when compared with PBA and BMS. Consequently, the National Institute for Health and Care Excellence (NICE) and the National Institute for Health Research's Health Technology Assessment (HTA) programme both recommended that a randomised controlled trial (RCT) be conducted in patients with SLI to establish whether these new drug technologies confer additional clinical benefit and represent a cost-effective use of the available NHS resources [5, 9].

The BASIL-3 trial directly addresses the HTA call (13/81) as a RCT in which patients with SLI secondary to atherosclerotic femoro-popliteal (FP) disease \pm infrapopliteal (IP) disease are randomly allocated to: $\mathrm{PBA} \pm$ bail-out BMS, $\mathrm{DCB} \pm$ bail-out BMS, or primary DES as their first revascularisation strategy (see Figs. 1 and 2 for the BASIL-3 Consolidated Standards of Reporting Trials (CONSORT) and Standard Protocol Items: Recommendations for Interventional Trials (SPIRIT) diagrams, respectively. A SPIRIT Checklist is also provided as Additional file 1). BASIL-3 includes an internal pilot phase and health economic analysis. The primary clinical outcome is amputation-free survival (AFS) defined as the time to major (above the ankle) amputation of the index limb or death from any cause, whichever occurs first; secondary outcome measures are shown in Table 1 . The primary outcome for the costeffectiveness analysis is cost per quality-adjusted life year (QALY) gained.

\section{Methods \\ Indemnity}

This is a clinician-initiated study. The sponsor (University of Birmingham) holds public liability (negligent harm) and clinical trial (negligent harm) insurance policies which apply to this trial. Full details of indemnity arrangements for this trial can be obtained from the sponsor.

\section{Eligibility}

Full inclusion/exclusion criteria are shown in Table 2. All patients with SLI referred to vascular units within participating NHS organisations may be considered for enrolment (a full list of participating centres can be 


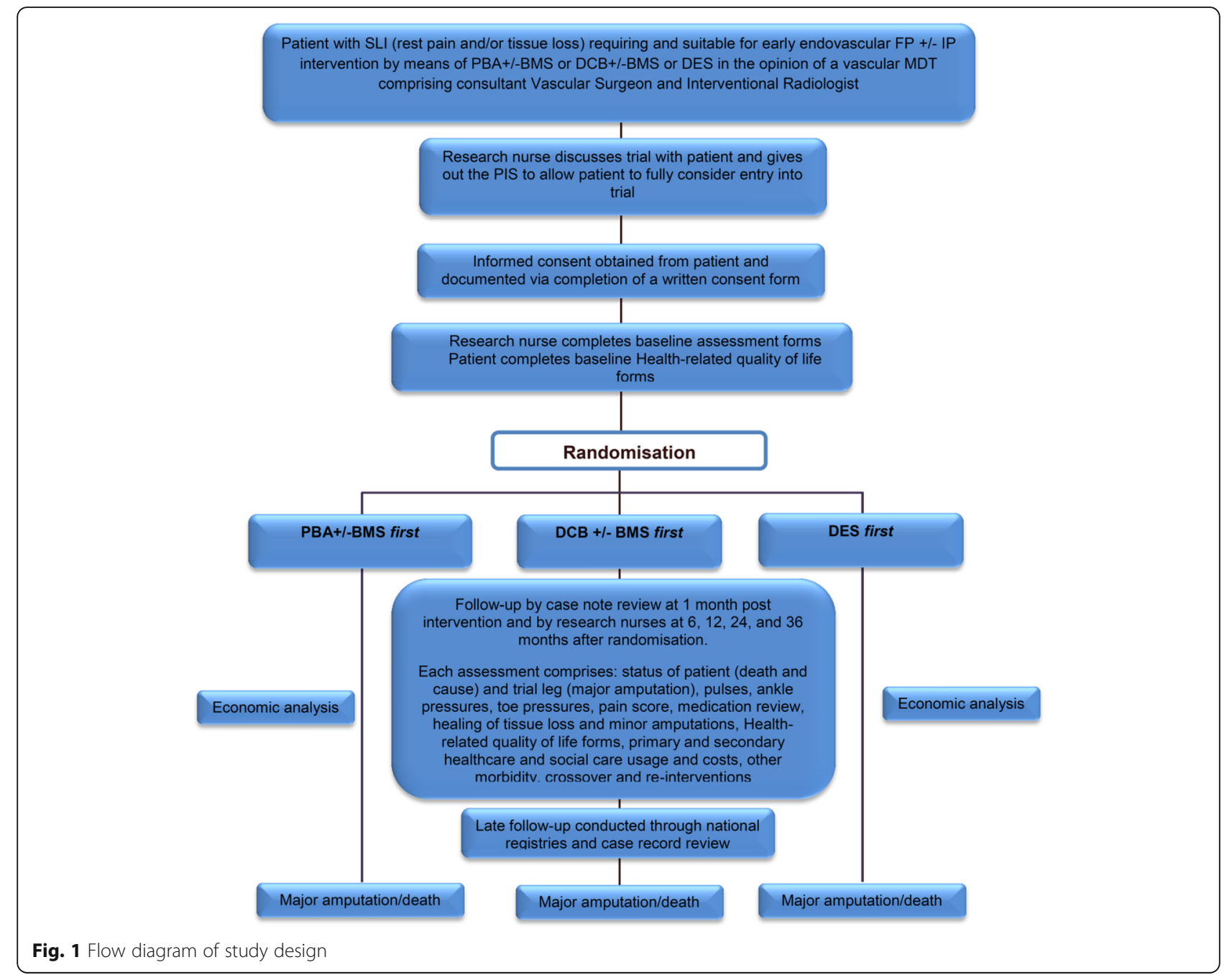

obtained from the trial sponsor). Participants should have atherosclerotic FP disease and be judged by the vascular multidisciplinary team (consisting of two endovascular practitioners from either vascular surgery or interventional radiology) to require early endovascular revascularisation. Following diagnostic imaging, all participants should be deemed suitable to receive any of the three trial revascularisation strategies and should also have adequate inflow to support these strategies (either at the time of randomisation or achieved as part of a 'hybrid' procedure in which inflow is restored concurrently with the FP revascularisation). Participants should have an anticipated life expectancy of at least 6 months and be able to complete the trial quality of life (QoL) and resource use questionnaires. Patients will be excluded from the trial if they lack capacity to provide written informed consent, have received a previous intervention to the target vessel within the past 12 months, or speak insufficient English to guarantee informed consent where translation services are not available.

\section{Randomisation}

Randomisation will be provided by a computer-generated programme hosted online from Birmingham Clinical Trials Unit (BCTU), using a minimisation algorithm with a random element incorporated within it to ensure balance between the three arms with regard to important clinical variables. The following minimisation variables will be used:

- Age $(\leq 60,61-70,71-80$, or $>80$ years $)$

- Sex (male or female)

- DM (presence or absence)

- CKD (presence or absence of stage 3 or higher)

- Severity of clinical disease (ischaemic rest pain only, tissue loss only, or both)

- Artery being treated (superficial femoral, popliteal, or both)

- Recruiting clinical centre

- Prior permissible intervention to the trial leg, defined as an intervention to the target vessel more 


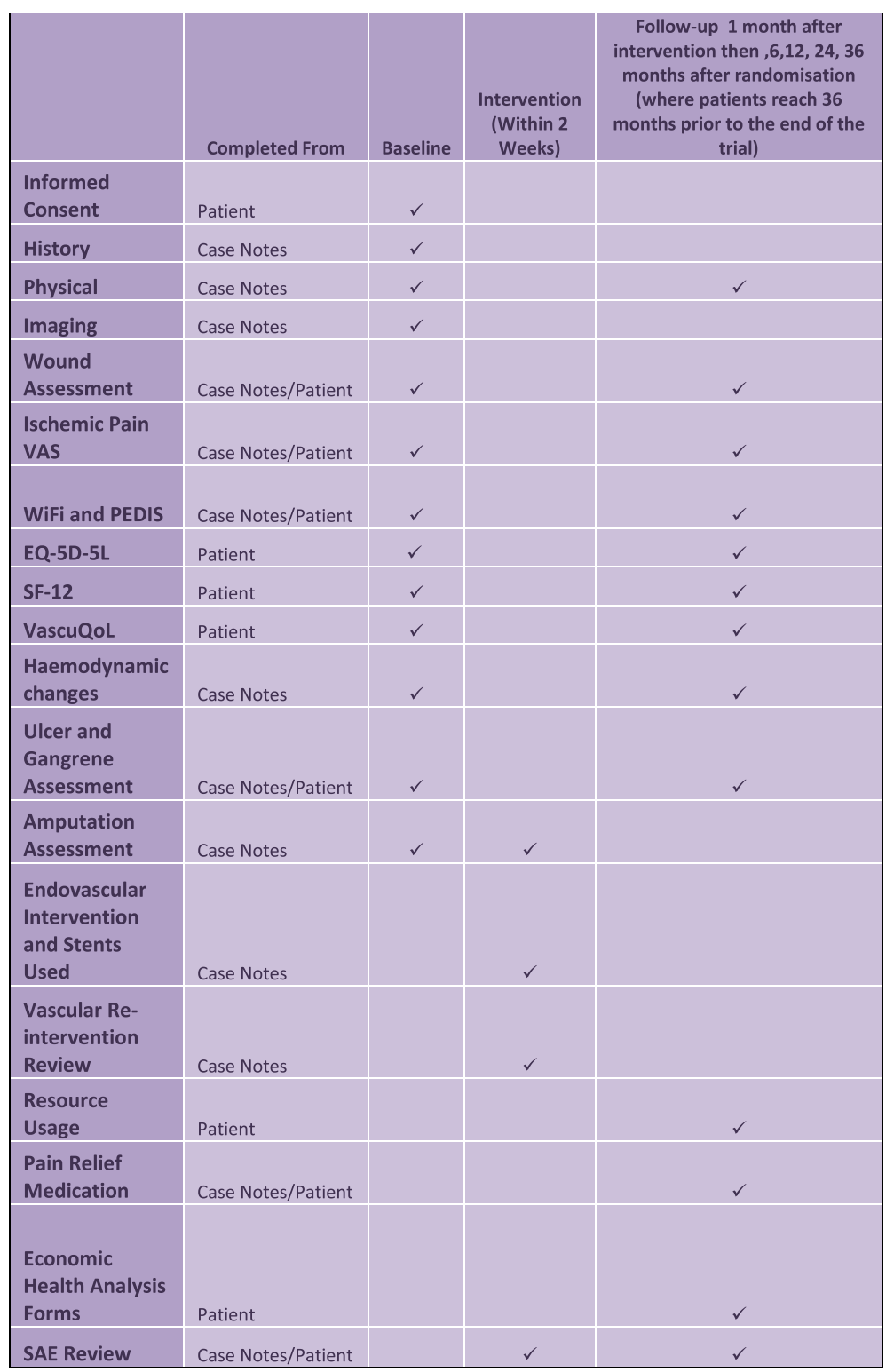

Fig. 2 Standard Protocol items: Recommendations for Interventional Trials (SPIRIT) diagram

than 12 months ago or to a different vessel in the trial leg at any time (yes or no)

- Hybrid procedure planned (yes or no)

Randomisation will be performed between the three endovascular interventions at a ratio of 1:1:1.

\section{Baseline assessment}

All participants will undergo a baseline assessment consisting of:

1. Patient's medical history including cardiovascular risk factors, comorbidities, functional status as well as prior vascular interventions and amputations to both legs

2. Clinical status comprising peripheral pulses, anklebrachial pressure index (ABPI), toe-brachial pressure index ((TBPI) where indicated), and current drug therapy

3. Foot assessment using the "Wound, Ischaemia, and Foot Infection' (WiFi) and 'Perfusion Extent Depth Ischaemia Sensation' (PEDIS) tools $[10,11]$

4. Review of investigations including diagnostic imaging.

5. Assessment of patient's QoL by use of the following tools:

a. European quality of life 5 level score (EQ-5D-5L) [12] 
Table 1 Secondary outcome measures

\section{Overall survival}

Major adverse limb events (MALE), defined as amputation (transtibial or above) or any major vascular re-intervention (thrombectomy, thrombolysis, PBA, stenting, or surgery)

In-hospital and 30-day morbidity and mortality

Major adverse cardiac events (MACE), defined as SLI and amputation affecting the contralateral limb, ACS, or stroke

Relief of ischaemic pain (VAS, medication usage)

Psychological morbidity (using HADS)

Quality of life using generic (EQ-5D-5L, ICECAP-O, SF-12) and disease-specific (VascuQoL) tools

Re- and cross-over intervention rates

Healing of tissue loss (ulcers, gangrene) as assessed by the PEDIS and the WIFI scoring and classification systems

Extent and healing of minor (toe and forefoot) amputations (also using PEDIS and WIFI)

Haemodynamic changes; absolute ankle and toe pressures, ABPI, TBPI

$A B P I$ ankle-brachial pressure index, $P B A$ plain balloon angioplasty, EQ-5D-5L European quality of life 5 level score, HADS Hospital Anxiety and Depression Scale, ICECAP-O ICEpop CAPability measure for Older people, PEDIS Perfusion Extent Depth Ischaemia Sensation, SF-12 Short form-12 health survey version 2, TBPI toe-brachial pressure index, VAS Visual Analogue Scale, WIFI Wound, Ischaemia, and Foot Infection tool

b. ICEpop CAPability measure for Older people (ICECAP-O) [13]

c. Short form-12 health survey version 2 (SF-12) [14]

d. Visual Analogue Scale pain score (VAS) [15]

e. Hospital Anxiety and Depression Scale (HADS) [16]

f. Vascular quality of life (VascuQoL) [17]

\section{Trial intervention}

The randomised trial intervention should normally take place within 14 days of randomisation where clinically and logistically practical. The randomised endovascular procedure will normally be carried out under local anaesthesia with access via the common femoral artery. Technical success of the procedure will be assessed by pulse status, completion angiography, and haemodynamic measures such as ABPI. Data collected relating to the randomised procedure will include type and number of the devices used by artery segment as well as disease severity. Standard data relating to the hospital admission or day case will be collected and include summary of the admission, discharge destination, additional interventions, and medical complications.

\section{Repeat and cross-over interventions}

Further intervention is possible in all arms of the trial, even when the trial endovascular intervention has been successful (for example, due to multilevel disease or following restenosis). This may either be with the same endovascular intervention (re-intervention), one of the alternative endovascular interventions (endovascular cross-over intervention) or surgical intervention (surgical cross-over intervention), each of which may be repeated more than once.

Based on clinical experience, and data from the original BASIL trial [18], we anticipate that further intervention:

- Will be required in up to $20 \%$ of participants

- Is most likely to be required within 12 months of randomisation

\section{Follow-up}

Outcomes will be recorded at 1 month post intervention and 6, 12, 24, and 36 months post randomisation. Data collected will include peripheral pulses, ABPI, TBPI (where indicated), functional status, and current drug therapy. All participants will undergo a full review of hospitalisations, further interventions (vascular, nonvascular, and amputations), and (serious) adverse events (SAEs/AEs). The QoL assessments (previously described) will be completed at each follow-up time point as well as health resource usage and personal circumstance assessments as part of the economic analysis. A schedule of assessments for all time points is given in Fig. 2.

Table 2 Inclusion and exclusion criteria

\begin{tabular}{ll}
\hline Inclusion criteria & Exclusion criteria \\
\hline Have SLI due to atherosclerotic FP, \pm IP, PAD & Have an anticipated life expectancy $<6$ months \\
$\begin{array}{l}\text { Be judged by the responsible clinicians (consultant VS, IR) working as } \\
\text { part of a MDT to: Require early FP, } \pm \text { IP, endovascular revascularisation } \\
\text { in addition to BMT, foot and wound care }\end{array}$ & Be, in the opinion of the clinician, unable to provide informed consent \\
$\begin{array}{l}\text { Have or will have adequate 'inflow' to support all trial revascularisation } \\
\text { strategies }\end{array}$ & $\begin{array}{l}\text { Be a non-English speaker where local translation facilities are insufficient } \\
\text { to guarantee informed consent }\end{array}$ \\
$\begin{array}{l}\text { Judged suitable for all trial revascularisation strategies following } \\
\text { diagnostic imaging and a documented MDT discussion }\end{array}$ & $\begin{array}{l}\text { Be judged unsuitable for the endovascular revascularisation strategies by } \\
\text { a vascular MDT }\end{array}$ \\
Able to complete QoL and resource use booklet & $\begin{array}{l}\text { Previous intervention (BET or bypass) to the target vessel within the } \\
\text { past 12 months }\end{array}$ \\
\hline
\end{tabular}

$B E T$ best endovascular therapy BMT best medical therapy IP infrapopliteal FP femoro-popliteal MDT multi-disciplinary team PAD peripheral arterial disease, QoL quality of life SLI severe limb ischaemia 
Appropriate strategies to promote participant retention will be considered and implemented by the Trial Management Group (TMG) as required.

Outcomes will be collected by research nurses at the clinical centres on paper Case Report Forms (CRFs). All data will be handled in accordance with the UK Data Protection Act, 1998. CRFs, other than the Patient Contact Form and Consent Form, will not bear the participant's name. For all other forms the participant's initials, date of birth and trial number will be used for identification.

\section{Safety reporting}

The collection and reporting of AEs and SAEs will be in accordance with Good Clinical Practice (GCP) and the Research Governance Framework 2005. Safety will be assessed continuously throughout the trial. There are no investigational medicinal products being used as part of BASIL-3 and all of the surgical procedures being tested in this trial are part of current UK 'standard of care'; therefore, no (S)AEs are anticipated as a unique consequence of participation in BASIL-3. In addition, at regular time points, the Trial Steering Committee (TSC) and Data Monitoring Committee (DMC) will be provided with details of all SAEs.

\section{Sample size calculation}

The sample size for this trial was calculated based on a time-to-event analysis making two key comparisons between standard care and the new treatments (PBA \pm $\mathrm{BMS}$ versus $\mathrm{DES}$; and $\mathrm{PBA} \pm \mathrm{BMS}$ versus $\mathrm{DCB} \pm \mathrm{BMS})$. To maintain an overall $5 \%$ type I error rate, each comparison will be tested at a significance level of $2.5 \%$ to account for the increase in the risk of type I error associated with making two key comparisons. The total trial duration is 5 years with 3 years' recruitment $(20 \%$ of participants are to be recruited in year 1 , and $40 \%$ in years 2 and 3 ) and 2 years' follow-up resulting in a mean follow-up of 3.3 years per patient.

The sample size calculation is based on estimated event rates in the $\mathrm{PBA} \pm \mathrm{BMS}$ arm taken from the angioplasty arm of the original BASIL trial (observed to be $0.70,0.64,0.52,0.46$, and 0.36 at the end of years $1-5$, respectively) [18]. The study is powered at $90 \%$ to detect a hazard ratio of 0.60 for both comparisons reducing the risk of the primary outcome (AFS). Across the three arms, a total of 342 events would be required to detect a hazard ratio of 0.60 (equivalent to an absolute difference in AFS of $13 \%$ at year 2) at the $2.5 \%$ significance level. Conservatively, allowing for $5 \%$ drop out for the primary outcome (equivalent to $1 \%$ dropout in each year for 5 years) a total of 861 participants is required. It is anticipated that around 50 UK clinical centres will be required to achieve this recruitment target. Appropriate strategies to meet recruitment targets will be discussed and implemented by the TMG as required.

\section{Pilot phase}

Recruitment to BASIL-3 will be reviewed at the end of a 12-month pilot phase and discontinuation of the trial will be considered if:

- Fewer than 15 clinical centres have been opened

- Fewer than two out of three opened centres have recruited a participant

- Fewer than 100 participants have been randomised

- Fewer than $80 \%$ of participants have received their allocated treatment

The completeness of the QoL measures will also be evaluated at the end of the pilot phase. If completion rates are poor, consideration will be given to discontinuing some of these tools.

\section{Data monitoring and interim analysis}

After the first year of recruitment, we aim to assess recruitment, retention, patient burden, and completeness of data. A full efficacy and safety analysis report will be reviewed by the DMC on an annual basis or more frequently if required by the DMC or TMG. The DMC will outline and agree the stopping rules for the trial which will be documented in the DMC charter. It is likely that the Haybittle-Peto boundary will be used. This approach states that if an interim analysis of the primary outcome shows, with a $p$ value of less than 0.001 , that the treatments are different, then the trial should be stopped early. This Haybittle-Peto approach will be used as stopping guide, alongside data on important secondary endpoints and all other relevant evidence. A DMC report and charter outlining the terms of reference (including information on stopping rules) will be agreed with the DMC. The report will specify which endpoints are to be included in the reports to the TSC. The membership of the DMC is stated within the current trial protocol which is published online [19].

Monitoring of BASIL-3 will ensure compliance with GCP. A risk proportionate approach to the initiation, management and monitoring of BASIL-3 will be adopted. Clinical centres will permit trial-related monitoring, audits, and Main Research Ethics Committee review; providing direct access to source data/documents. Trial participants will be informed of this during the informed consent discussion and will consent to provide access to their medical notes for these purposes. Important changes to the trial conduct or trial protocol will be communicated to all relevant interested parties and the trial sponsor assumes responsibility for this. 


\section{Final analysis}

The final analysis for the BASIL-3 trial will occur once the last randomised patient reaches the 24-month follow-up assessment. Differences in the primary outcome (AFS) will be assessed by comparing time from randomisation to major limb amputation or death from any cause between randomised groups, assessed up until the end of the follow-up period which will be between 24 and 60 months.

The primary unadjusted analysis will use KaplanMeier plots and test the difference between groups using the log-rank test. Data will be censored when individuals reach the end of follow-up or are lost to follow-up prior to reaching the primary outcome. Further analysis of the primary outcome will involve fitting flexible parametric survival models to estimate both the relative and absolute differences in the hazard of the primary outcome, to model the underlying differences in hazard, and to allow for nonproportional hazards. Addition of covariates to this model will allow adjustment for any baseline differences, and the addition of their interactions with the treatment allocation variable will test for subgroup effects. These models will allow examination of differences in effect for short-, medium-, and longer-term follow-up. The primary analysis of AFS will be undertaken on an intention-to-treat basis according to allocated first intervention, regardless of whether the intervention was delivered and whether repeat and cross-over interventions were subsequently undertaken.

Secondary outcome measures that are based on a continuous scale will be analysed using a repeated measures, multilevel model to examine any differential effect over time. Where necessary, data transformations will be made to fulfil modelling assumptions. Treatment effects from the repeated measures model will be reported at the 1month, 12-month, and 'end of follow-up' time points.

Other outcome measures will be explored using standard methods (Fisher's Exact Test for dichotomous outcomes, log-rank test for time to event data) and will also be reported at 1 month, 12 months, and at the end of follow-up.

As the pattern of repeat and cross-over procedures is likely to be multiple and complex, these will be measured as outcomes and no attempt will be made to adjust for them. Effect sizes will be presented as point estimates, $95 \%$ confidence intervals, and associated $p$ values.

\section{Sub-group analysis}

Variation in the treatment effect between subgroups will be limited to prespecified variables and investigated using appropriate tests for interaction in survival and repeated measures models. Variables likely to be considered will include, but will not necessarily be restricted to, ischaemic rest/night pain only versus tissue loss, or both rest pain and tissue loss, presence of DM, and presence of CKD.

\section{Economic analysis}

The economic analysis will comprise both 'within-study' and 'model-based' analyses. The 'within-study' analysis will be carried out to determine the cost-effectiveness of all three trial-mandated interventions on the basis of the patient-level data obtained during the study period.

Costs will take into account the use of NHS resources related to the primary interventions and any secondary procedures, readmissions, SAEs, hospital stays, day-case admissions, outpatient visits, and appointments with general practitioners and nurses. Changes in healthrelated QoL and QALYs will be calculated according to patients' responses to the EQ-5D-5L instrument. Results of the analysis will be presented in terms of cost per year of AFS and cost per additional QALY gained. In line with existing recommendations, the base-case analysis will adopt a health care system (payer's) perspective by considering costs incurred by the NHS and Personal Social Services [20].

The model-based analysis will be conducted to extend the within-study evaluation by considering costs and benefits likely to accrue beyond the study follow-up period. This will be based on a decision analytic model which will be built to serve as a framework for quantifying long-term costs and outcomes.

\section{Dissemination policy}

The chief investigator will coordinate dissemination of data from BASIL-3. All publications and presentations, including abstracts, relating to the main trial will be authorised by the BASIL-3 TMG. The results of the analyses will be published in the name of the BASIL-3 Collaborative Group in peer-reviewed journals (provided that this does not conflict with the journal's policy). All contributors to the trial will be listed, with their contribution identified. Trial participants will be sent a summary of the final results of the trial which will contain a reference to the full papers. All applications from groups wanting to use BASIL-3 data to undertake original analyses will be submitted to the TMG for consideration before release. To safeguard the scientific integrity of BASIL-3, trial data will not be presented in public before the main results are published without the prior consent of the TMG.

\section{Discussion}

BASIL-3 is a pragmatic RCT which is designed to inform future UK 'standard of care' for patients presenting with SLI secondary to FP disease. Many of these patients will have multilevel disease including both 'inflow' (aorto-iliac) disease as well as 'outflow' or 'below the knee' (IP) disease. In such patients it will be permissible to perform additional interventions in accordance with routine practice at the same time as the randomised FP 
intervention. Moreover, it would also be permissible to restore inflow by performing an aorto-iliac intervention during an initial procedure and then randomise for an endovascular FP intervention at a later date if this is still deemed to be appropriate.

Enrolment into the BASIL-3 trial is on a per-patient basis and only one leg may be randomised into the trial. However, data from the original BASIL trial suggest that up to a quarter of patients may present with bilateral SLI, in which case the 'worst' leg (which is usually clinically obvious) should be entered as the trial limb.

\section{Trial status}

At the time of writing, the BASIL-3 trial was halfway through the study's pilot phase with 15 clinical centres open to recruitment and over 50 participants randomised.

\section{Additional file}

Additional file 1: BASIL-3 SPIRIT Checklist. (DOC $122 \mathrm{~kb}$ )

\section{Abbreviations}

ABPI: Ankle-brachial pressure index; AE: Adverse event; AFS: Amputation-free survival; BCTU: Birmingham Clinical Trials Unit; BMS: Bare metal stent; CKD: Chronic kidney disease; CRF: Case Report Form; DCB: Drug-coated balloon; DES: Drug-eluting stent; DM: Diabetes mellitus; DMC: Data Monitoring Committee; DMC: Data Monitoring Committee; FP: Femoropopliteal; HTA: Health Technology Assessment Programme; IP: Infrapopliteal; ITT: Intention-to-treat; NICE: National Institute for Health and Care Excellence; NIHR: National Institute for Health Research; PAD: Peripheral arterial disease; PBA: Plain balloon angioplasty; PEDIS: Perfusion Extent Depth Ischaemia Sensation tool; QALY: Quality-adjusted life year; QoL: Quality of life; RCT: Randomised controlled trial; SAE: Serious adverse event; SLI: Severe limb ischaemia; TBPI: Toe-brachial pressure index; TSC: Trial Steering Committee; UK: United Kingdom; WIFI: Wound, Ischaemia, and Foot Infection tool

\section{Acknowledgements}

Not applicable.

\section{Funding}

This project was funded by the National Institute for Health Research (project number 13/81/02). The funder approved the final trial protocol prior to its implementation.

\section{Availability of data and materials}

The datasets analysed during and/or after this study will be made available from the corresponding author on reasonable request and upon approval from the trial sponsor.

\section{Authors' contributions}

All authors have read and approved the final version of this manuscript. $\mathrm{BH}$ is the trial manager and was responsible for the preparation and drafting of the manuscript. MP assisted with manuscript writing and proof reading. HD assisted with manuscript writing and proof reading. LM assisted with manuscript writing and proof reading. HJ assisted with manuscript writing and proof reading. GB is the lead research nurse for the trial and assisted in proof reading the manuscript. MG is the operations manager of the Clinical Trials Unit and assisted in proof reading the manuscript for accuracy. SP provided statistical assistance including critical review of the statistics section. $\mathrm{CH}$ provided statistical assistance including critical review of the statistics section. LA is the lead health economist working on the trial. He designed the economic component of the BASIL study protocol and authored the 'Economic analysis' section of this manuscript. JD is the director of the Clinical Trials Unit and is responsible for critical review of the manuscript. AB is the trial chief investigator and is responsible for trial conception, design, and final approval of this manuscript.

\section{Authors' information}

Benjamin Hunt (BH) BMedSc (Hons), PhD, Clinical Trial Coordinator, Birmingham Clinical Trials Unit

Matthew Popplewell (MP) MBChB, MRCS, Vascular Research Fellow, Birmingham University Department of Vascular Surgery

Huw Davies (HD) MBBS, BSc, MRCS, Vascular Research Fellow, Birmingham University Department of Vascular Surgery

Lewis Meecham (LM) MBBCH, MRCS, Vascular Research Fellow, Birmingham University Department of Vascular Surgery

Hugh Jarrett $(\mathrm{HJ})$ BSc, BA, MSc, Senior Trial Coordinator and Team Leader, Birmingham Clinical Trials Unit

Gareth Bate (GB) RGN, PGCert, Lead Research Nurse, Birmingham University Department of Vascular Surgery

Margaret Grant (MG) PhD, Operations Manager, Birmingham Clinical Trials Unit Smitaa Patel (SP) BSC, MSc, Senior Statistician, Birmingham Clinical Trials Unit Catherine Hewitt $(\mathrm{CH})$ BSC, MSc, Statistician, Birmingham Clinical Trials Unit Lazaros Andronis (LA) BSc, MSc, PhD, Lecturer in Health Economics, Health Economics Unit, University of Birmingham

Jonathan Deeks (JD) BSc, MSc, PhD, CStat, Professor/Director, Birmingham Clinical Trials Unit

Andrew Bradbury (AB), BSC, MBChB (Hons), MBA, MD, FEBVS, FRCSEd,

FRCSEng, Chief Investigator, Birmingham University Department of Vascular Surgery

\section{Competing interests}

The authors declare that they have no competing interests.

\section{Consent for publication}

Not applicable.

\section{Ethics approval and consent to participate}

Ethical approval was granted from the National Research Ethics Service, North of Scotland, on 26 August 2015 (reference 15/NS/0070). All trial participants will provide full written informed consent prior to randomisation. Written informed consent will be sought by individuals who hold a substantive contract of employment or honorary contract with the appropriate NHS organisation and who are delegated to do so by the principal investigator at that clinical centre. Copies of the Patient Information Leaflet and Consent Form for this trial are available from the trial website [19].

\section{NIHR disclaimer}

This paper presents independent research funded by the National Institute for Health Research (NIHR). The views expressed are those of the author(s) and not necessarily those of the NHS, the NIHR, or the Department of Health.

\section{Publisher's Note}

Springer Nature remains neutral with regard to jurisdictional claims in published maps and institutional affiliations.

\section{Author details}

${ }^{1}$ Birmingham Clinical Trials Unit, Institute of Applied Health Research, College of Medical and Dental Sciences, Public Health Building, University of Birmingham, Birmingham B15 2TT, UK. ${ }^{2}$ Heart of England NHS Foundation Trust, Netherwood House, Solihull Hospital, University Department of Vascular Surgery, Lode Lane, Solihull B91 2JL, UK. ${ }^{3}$ Health Economics Unit, Institute of Applied Health Research, Public Health Building, University of Birmingham, Birmingham B15 2TT, UK.

Received: 16 September 2016 Accepted: 4 May 2017

Published online: 19 May 2017

\section{References}

1. Shammas NW. Epidemiology, classification, and modifiable risk factors of peripheral arterial disease. Vasc Health Risk Manag. 2007;3(2):229-34.

2. Abu Dabrh AM, Steffen MW, Undavalli C, Asi N, Wang Z, Elamin MB, Conte MS, Murad MH. The natural history of untreated severe or critical limb ischemia. J Vasc Surg. 2015;62(6):1642-51. e1643. 
3. Norgren L, Hiatt WR, Dormandy JA, Nehler MR, Harris KA, Fowkes FG, Group TIW. Inter-society consensus for the management of peripheral arterial disease (TASC II). J Vasc Surg. 2007:45 Suppl S:S5-67.

4. Varu VN, Hogg ME, Kibbe MR. Critical limb ischemia. J Vasc Surg. 2010; 51(1):230-41.

5. National Institute for Health and Clinical Excellence. Lower limb peripheral arterial disease. Diagnosis and management. NICE clinical guideline 147. In National Clinical Guideline Centre. 2012.

6. Swaminathan A, Vemulapalli S, Patel MR, Jones WS. Lower extremity amputation in peripheral artery disease: improving patient outcomes. Vasc Health Risk Manag. 2014;10:417-24.

7. Diamantopoulos A, Katsanos K. Treating femoropopliteal disease: established and emerging technologies. Semin Interv Radiol. 2014;31(4):345-52.

8. Pastromas G, Katsanos K, Krokidis M, Karnabatidis D, Spiliopoulos S. Emerging stent and balloon technologies in the femoropopliteal arteries. ScientificWorldJournal. 2014;2014:695402.

9. NIHR Journals Library: 13/81/02. http://www.nets.nihr.ac.uk/projects/hta/ 138102. Accessed 13 July 2016.

10. Karthikesalingam A, Holt PJ, Moxey P, Jones KG, Thompson MM, Hinchliffe RJ. A systematic review of scoring systems for diabetic foot ulcers. Diabet Med. 2010;27(5):544-9.

11. Mills Sr JL, Conte MS, Armstrong DG, Pomposelli FB, Schanzer A, Sidawy AN, Andros G, Society for Vascular Surgery Lower Extremity Guidelines C. The Society for Vascular Surgery lower extremity threatened limb classification system: risk stratification based on wound, ischemia, and foot infection (WIfI). J Vasc Surg. 2014;59(1):220-34. e221-222.

12. Herdman M, Gudex C, Lloyd A, Janssen M, Kind P, Parkin D, Bonsel G, Badia $X$. Development and preliminary testing of the new five-level version of EQ5D (EQ-5D-5L). Qual Life Res. 2011;20(10):1727-36.

13. Flynn TN, Chan P, Coast J, Peters TJ. Assessing quality of life among British older people using the ICEPOP CAPability (ICECAP-O) measure. Appl Health Econ Health Policy. 2011;9(5):317-29.

14. Jenkinson C, Layte R. Development and testing of the UK SF-12 (short form health survey). J Health Serv Res Policy. 1997;2(1):14-8.

15. Wewers ME, Lowe NK. A critical review of visual analogue scales in the measurement of clinical phenomena. Res Nurs Health. 1990:13(4):227-36

16. Zigmond AS, Snaith RP. The Hospital Anxiety and Depression Scale. Acta Psychiatr Scand. 1983:67(6):361-70.

17. Morgan MB, Crayford T, Murrin B, Fraser SC. Developing the Vascular Quality of Life Questionnaire: a new disease-specific quality of life measure for use in lower limb ischemia. J Vasc Surg. 2001;33(4):679-87.

18. Bradbury AW, Adam DJ, Bell J, Forbes JF, Fowkes FG, Gillespie I, Ruckley CV, Raab GM, Participants B. Bypass versus Angioplasty in Severe Ischaemia of the Leg (BASIL) trial: an intention-to-treat analysis of amputation-free and overall survival in patients randomized to a bypass surgeny-first or a balloon angioplasty-first revascularization strategy. J Vasc Surg. 2010;51(5 Suppl):5S-17.

19. BASIL-3 trial website. www.birmingham.ac.uk/basil3. Accessed 13 July 2016.

20. Guide to the methods of technology appraisal (PMG9). https://www.nice. org.uk/process/pmg9/chapter/foreword. Accessed 13 July 2016.

\section{Submit your next manuscript to BioMed Central and we will help you at every step:}

- We accept pre-submission inquiries

- Our selector tool helps you to find the most relevant journal

- We provide round the clock customer support

- Convenient online submission

- Thorough peer review

- Inclusion in PubMed and all major indexing services

- Maximum visibility for your research

Submit your manuscript at www.biomedcentral.com/submit
Biomed Central 